\title{
La ingeniería didáctica en la enseñanza de la noción del número en Educación primaria
}

\author{
Didactic engineering in teaching the notion of the number in Primary Education
}

HERNÁNDEZ-GUTIÉRREZ, Francisco Javier† \& SILVA-ALFARO, Andrea Monserrath

Escuela Normal Rural “Gral. Matías Ramos Santos” Secretaría de Educación del Estado de Zacatecas. Av José Santos Valdéz 6, 98820 San Marcos, Zac.

ID $1^{\mathrm{er}}$ Autor: Francisco Javier, Hernández-Gutiérrez / ORC ID: 0000-0002-4134-134, Researcher ID: L-2003-2017, CVU CONACYT ID: 428448

ID $1^{\text {er }}$ Coautor: Andrea Monserrath, Silva-Alfaro / ORC ID: 0000-0001-7866-6812, CVU CONACYT ID: 1011452

DOI: $10.35429 / J B E .2019 .7 .3 .16 .26$

Recibido 10 de Enero, 2019; Aceptado 18 de Marzo, 2019

\section{Resumen}

El presente trabajo es un artículo que se desprende de un trabajo más general de investigación educativa. Corresponde al paradigma de investigación cualitativo y de tipo investigación-acción. Es desarrollada en el contexto de la educación primaria con alumnos de primer grado. Tiene como principal objetivo conocer las implicaciones del conocimiento del contenido matemático y didáctico en la enseñanza aprendizaje de noción del número, para contar con las herramientas necesarias que permitan aplicar una metodología apropiada para su construcción. La metodología corresponde a la ingeniería didáctica. Considera una indagación previa sobre investigaciones referentes al tema que se aborda. Se analizan desde el aspecto teórico y metodológico con base en ideas centrales de autores importantes para este tema de estudio que a su vez se contrasta con los resultados obtenidos. Los resultados finales y conclusiones resaltan que la preparación del docente en las implicaciones conceptuales y didácticas, son base fundamental para que se desarrolle la construcción del número: conceptualmente, los términos y principios relacionados con las operaciones cognitivas de clasificación y seriación; didácticamente, corresponde a la formulación de situaciones problemas, orientado a una interacción de momentos y relaciones didácticas. Todo esto, llevado a cabo conforme los momentos de la ingeniería didáctica.

Concepto del número, Ingeniería didáctica, Didáctica de las matemáticas

\begin{abstract}
This paper is an article that emerges from a more general educational research work. It corresponds to the paradigm of qualitative research and action research type. It is developed in the context of primary education with first graders. Its main objective is to know the implications of the knowledge of the mathematical and didactic content in the teaching of the notion of the number, to have the necessary tools to apply an appropriate methodology for its construction. The methodology corresponds to didactic engineering. It considers a previous inquiry about research related to the topic being addressed. They are analyzed from the theoretical and methodological aspect based on central ideas of important authors for this subject of study, which in turn contrasts with the results obtained. The final results and conclusions highlight that the teacher's preparation in the conceptual and didactic implications is a fundamental basis for the construction of the number: conceptually, the terms and principles related to the cognitive operations of classification and seriation; didactically, corresponds to the formulation of problem situations, oriented to an interaction of moments and didactic relationships. All this, carried out according to the moments of didactic engineering.
\end{abstract}

Number concept, Didactic engineering, Mathematics teaching

Citación: HERNÁNDEZ-GUTIÉRREZ, Francisco Javier \& SILVA-ALFARO, Andrea Monserrath. La ingeniería didáctica en la enseñanza de la noción del número en Educación primaria. Revista de Educación Básica. 2019. 3-7: 16-26.

$\dagger$ Investigador contribuyendo como primer autor. 


\section{Introducción}

El presente trabajo de investigación busca dilucidar la relación con los aspectos que el docente de educación primaria necesita para desarrollar una práctica educativa favorable desde las perspectivas epistemológico, didácticas en la asignatura de matemáticas, de manera específica en el proceso por el que transitan los alumnos de los primeros años escolares con la noción del número, bajo la propuesta metodológica de una Ingeniería Didáctica. Corresponde a la línea de generación y divulgación del conocimiento del cuerpo académico en consolidación "El conocimiento especializado del profesor de matemáticas" de la Escuela Normal Rural "Gral. Matías Ramos Santos" de San Marcos, Loreto, Zacatecas y que está registrada en el Programa de Mejoramiento del Profesorado (PROMEP) de la Secretaria de Educación Pública.

\section{Planteamiento del problema}

El aprendizaje de las matemáticas en la escuela primaria es un tema que se encuentra vigente y que no deja de ser importante por la complejidad que tiene, tanto para los docentes, como para los alumnos. Esa complejidad aunada con las características de los alumnos, el contexto, el conocimiento didáctico y disciplinar de las matemáticas, etc., son aspectos que tienen mucho que ver dentro de este proceso y por ello, es preciso retomarlos en estudios sistemáticos.

Cuando se habla de la adquisición del concepto del número, también se habla de un proceso por el que pasan los alumnos más pequeños de la educación primaria. Aunque este proceso inicia desde el nivel preescolar, el proceso de aprendizaje de cada alumno es diferente, y es ahí donde el docente interviene para potencializar esos aprendizajes y el logro de tal proceso. En este caso, el mayor peso lo tienen las implicaciones de dominio del contenido matemático y su didactificación, que el docente debe gestionar para sus alumnos, a partir de una metodología que le permita el desarrollo de competencias matemáticas y alcance la conceptualización consciente del número.
Al focalizar la mirada en este punto de las implicaciones del dominio del contenido matemático y su didáctica para la concepción del número, consiste por un lado, en conocer los conocimientos que el docente debe dominar en el tema del número; y por el otro, el conocimiento y desarrollo de la didáctica para la enseñanza del número, con una metodología, que en este caso, es la ingeniería didáctica.

El Programa de Estudios para Educación Primaria 2011 clarifica que "la formación matemática que permite a los individuos enfrentar con éxito los problemas de la vida cotidiana depende en gran parte de los conocimientos adquiridos y de las habilidades y actitudes desarrolladas durante la Educación Básica" (2011, pág. 75), por ello, los intereses que se tienen al realizar esta investigación estriban en las dificultades que los docentes tienen para llevar a los alumnos en el camino de este proceso. El proceso de enseñanzaaprendizaje de las matemáticas, en específico del número, es largo y complejo. Influyen un sinnúmero de aspectos para que pueda darse favorablemente en los alumnos. En el caso específico de los docentes, su preparación y desenvolvimiento en el aula, es uno de los aspectos trascendentales en los que se precisa hacer un análisis y reflexión. Es por esto, que se planteó la siguiente interrogante como punto central de la investigación:

¿Cuáles son las implicaciones en el conocimiento del contenido matemáticos y su didáctica en la enseñanza de la noción del número considerando como metodología de enseñanza la ingeniería didáctica?

Desde estas consideraciones, es preciso enmarcar los puntos de lo que se aspira lograr. A partir de la pregunta se diseñó un objetivo:

Conocer las implicaciones conceptuales y didácticas en la enseñanza aprendizaje del número en el proceso de su noción, para contar con las herramientas necesarias que permitan aplicar una metodología apropiada para su construcción.

Hipótesis de acción: 
La ingeniería didáctica como dispositivo de planificación en la enseñanza de la noción del número en los primeros grados de educación primaria permite al docente un mejor y mayor conocimiento del contenido y didáctica de las matemáticas y por lo tanto, un mejor aprendizaje por parte de los alumnos.

La investigación se llevó a cabo en un grupo de primer grado, que está integrado por 33 alumnos, de los cuales fueron 16 niñas y 17 niños. La ubicación del grupo es en la Escuela Primaria Urbana "Manuel Rangel Martínez", del municipio de Loreto, Zacatecas.

\section{Diseño de la investigación}

Hernández Sampieri, Fernández Collado \& Baptista Lucio (2006) mencionan que las investigaciones cualitativas se fundamentan más en un proceso inductivo (explorar y descubrir, y luego generar perspectivas teóricas), es de gran interés las interacciones entre los individuos, el proceso de indagación es flexible, es holística (considera el "todo"), tiene una perspectiva interpretativa enfocada en los humanos y sus instituciones, considera la realidad desde la mirada de los participantes y tiene un patrón cultural.

Además de esto, este tipo de investigación "proporciona profundidad a los datos, dispersión, riqueza interpretativa, contextualización del ambiente o entorno, detalles y experiencias únicas" (Hernández Sampieri, Fernández Collado, \& Baptista Lucio, 2006, pág. 21) lo que contribuye a que de manera conjunta con estos elementos, el proceso que realice sea circular, permitiendo que haya una interacción entre las etapas de la misma. Hernández Sampieri, Fernández Collado, \& Baptista Lucio (2006, pág. 523) también hace especifico que el proceso cualitativo no es lineal, sino iterativo o recurrente, las supuestas etapas en realidad son acciones para adentrarnos más en el problema de investigación, y es así que la orientación, permite que desde el punto de partida se consideren experiencias, el espacio y la interacción de los participantes, así como la recolección de datos. Es así que, reflexionando sobre las características, beneficios y oportunidades que permite el estudio de la investigación de tipo cualitativa, es la más conveniente para realizar este estudio.
Al hablarse de un área educativa y sobre el contexto de la educación básica (primaria), se ha planteado propicio llevarla a cabo. A partir de la investigación cualitativa que se presentan, de entre los diseños metodológicos que se puede adoptar (teoría fundamentada, etnografía, narrativa y de investigación-acción), se particulariza en el diseño basado en la investigación-acción, centrado como ya antes se había mencionado en el aspecto educativo. La investigación acción, es desde palabras de Elliott (1993) "un estudio de una situación social con el fin de mejorar la calidad de la acción dentro de la misma" (citado por Latorre, 2005, pág. 24) donde los problemas y procesos que lleva cabo el profesorado son los puntos base de su investigación y práctica.

La investigación acción cuenta con fases claras y precisas para llevarla a cabo: planificar, actuar, observar y reflexionar (Latorre, 2005, pág. 32), que son las que interactúan para llevar a cabo el espiral. Aunque particularmente se da más peso de entre las propuestas que menciona Latorre (2005, pág. 36) a la del modelo de Elliott que se compone de tres momentos base: elaborar un plan, ponerlo en marcha y evaluarlo; para luego rectificar el plan, ponerlo en marcha y evaluarlo y así sucesivamente, dentro de esta investigación se desarrolló de la siguiente manera:

Identificación de una idea general: a partir del interés particular de investigar sobre este tema, así como un diagnóstico especifico que caracterizó a los alumnos y docente, de manera que permitió centrar los objetivos de investigación.

Exploración o planteamiento de la hipótesis: recurrir a la indagación teórica metodológica, que orienten y conduzcan el tipo de actividades que se llevaron a cabo.

Construcción del plan de acción: diseño de una ingeniería didáctica contemplando tres sesiones de intervención en el aula.

Acción: llevar a cabo la ingeniería didáctica.

Evaluación: análisis a partir de un cuadro de triple entrada revisando fortalezas, debilidades y posibles cambios en las situaciones didácticas. 
Revisión del plan: planteamiento de aspectos de mejora de la practica conforme el análisis de fortalezas y debilidades.

Reconstrucción del plan: situación didáctica contemplando los aspectos de mejora con una intervención en el aula.

Acción: llevar a cabo la situación didáctica.

Evaluación: análisis específico de los aspectos de mejora, así como las situaciones que aún se encuentran con debilidades.

\section{Desarrollo y ejecución de la investigación. Ingeniería didáctica para la construcción de la noción del número}

La ingeniería que se desarrolló estuvo enmarcada bajo los momentos planteados por Michelle Artigue (1995) con el objetivo de poner en juego los conocimientos del contenido matemático y su didactificación para el proceso de la noción del número en los alumnos del primer grado. Los aprendizajes esperados que se pretenden enseñar correspondieron a contenidos diferentes, de manera que se distinga el cambio y avance.

\section{Análisis preliminar.}

La noción del número es constituida a partir de varios conceptos que trabajados en conjunto pueden definir el número como tal. Para comprender este conjunto de conceptos, desde algunas décadas atrás se ha preocupado por analizar el proceso a partir de las diferentes visiones pasando desde una conductista hasta lo que ahora tenemos como constructivista. Un caso significativo es el que presenta (Block \& Álvarez, 1999) donde precisamente revisa, compara y analiza cuatro décadas (60-90) bajo la asignatura de matemáticas, y más en específico con los números en primer grado. En la actualidad, Lizarde Flores (2016) hace una revisión del proceso de la matemática escolar que se trabaja en el plan actual y lo compara con los Materiales de Matemáticas para la educación Normal de tal forma que pudieron rescatar un paquete de conocimientos, visto como:
El grupo de temas que los profesores tienden ver alrededor del tema que enseñan; así, su articulación en un mapa conceptual permite apreciar las relaciones que se guarden entre si los diferentes contenidos: de complejización, de simplificación, transversales, o bien, auxiliares (Lizarde Flores, 2016, pág. 87)

En el proceso de construcción de la noción del número, se distingue que "se inicia con situaciones de conteo y gradualmente se va aumentando el rango de los números" (Lizarde Flores, 2016, pág. 90) de esta manera la complejidad va en aumento. También se destacan las habilidades requeridas para una mejor comprensión del contenido: "composición y descomposición, identificación, comparación, orden, lectura, escritura, agrupamiento, entre otros" (Lizarde Flores, 2016, pág. 90) y por otro el lado, son considerados "otros aspectos del contenido que contribuyen a su comprensión y fortalecimiento: por ejemplo los cortes didácticos en la formalización de saberes (conocimiento de los "nudos", valor posicional, la idea de sistema de numeración)", de este modo, bajo esos conocimientos y especialmente por la composición y descomposición, forma las bases para lograr llegar a la construcción del concepto de suma y resta que será el límite de consolidación del número en el que es posible y pertinente legar en el primer grado de educación primaria.

La enseñanza de la matemática en la escuela primaria.

La enseñanza que se llevó a cabo está constituida primordialmente por el Plan de estudios 2011, que encamina la intervención docente a partir de los principios pedagógicos enmarcados, y ponen al docente como una guía que facilita las herramientas para que el alumno sea quien construya su propio conocimiento. El actual enfoque está basado en competencias, pero bajo la premisa del constructivismo. A su vez, estas competencias para la vida son las que llevan a los alumnos a ser capaces de resolver "situaciones problemáticas que les plantea su vida y su entorno, a partir de la interrelación de elementos conceptuales, factuales, procedimentales y actitudinales para la toma de decisiones sobre la elección y aplicación de estrategias de actuación oportunas y adecuadas..." (SEP, 2011) 
Por su parte, el Programa de Estudios actual para primer grado (2011), en el campo formativo de pensamiento matemático, y como parte de la didáctica fundamental de las matemáticas menciona como finalidad el razonamiento para la resolución de problemas, la formulación de argumentos y el diseño de procedimientos o estrategias. Es por ello que la forma más conveniente que se ha considerado de planificar las clases es a partir de la Teoría de las Situaciones Didácticas, propuesta por Brousseau (2007), sus conceptos de: acción, formulación, validación, institucionalización, contrato didáctico, consigna, situación adidáctica, entre otros conducen a la solución de una situación problema para la construcción del conocimiento matemático.

\section{Concepción y análisis a priori}

El proceso didáctico en el que se presentaron las implicaciones conceptuales y didácticas de la noción del numeró de toda la investigación de la que se desprende este artículo, se organizaron en 4 sesiones clase que partieron de los momentos propuestos por la Teoría de las Situaciones Didácticas de Brousseau, empero para las intenciones de este artículo, se analizará los resultados de una de ellas.

\section{Clase 1.}

Competencias: Resolver problemas de manera autónoma. Comunicar información matemática. Validar procedimientos y resultados. Manejar técnicas eficientemente.

Intención didáctica: Que los alumnos resuelvan problemas que implican analizar la posición de las cifras de los números involucrados.

Contenido: Números y sistema de numeración: resolución de problemas que permitan iniciar el análisis del valor posicional de números con hasta dos cifras.

Preparación del medio, conocimientos previos y organización del grupo: Trabajo en binas con su compañero de banca, escribir en el pizarrón el número 12 y 89 preguntando ¿cuál de los dos es más grande? Y que comenten cómo saben cuál es el mayor.
Consigna, problema: La maestra Olga realizará una fiesta de cumpleaños, para la hora de la comida necesita poner 10 palillos de dientes en cada mesa. Tiene varios paquetes de palillos, pero ella sólo quiere el paquete que tenga más palillos.

Ayúdale a organizar los palillos en montones de 10 y registrarlos en la tabla para elegir el que tenga más.

\begin{tabular}{|l|c|c|}
\hline Número de palillos & $\begin{array}{c}\text { Montones } \\
\text { de 10 }\end{array}$ & $\begin{array}{c}\text { Palillos } \\
\text { sobrantes }\end{array}$ \\
\hline Paquete 1 & & \\
\hline Paquete 2 & & \\
\hline Paquete 3 & & \\
\hline Paquete 4 & & \\
\hline
\end{tabular}

\section{Tabla 1} palillos?

¿Cuál de los paquetes tiene más

¿Cuál tiene menos?

¿Cómo lo sabes?

Devolución de consigna: Preguntar a los alumnos sobre la situación que se ha planteado y sus datos, ¿qué le ocurre a la maestra Olga?, ¿cuál es el problema?, ¿qué es lo que van a hacer? En caso de tener alguna duda, solicitar a otro de los infantes que ayude en la resolución de la misma.

Restricciones: Pueden utilizar el material que crean necesario para contar. El tiempo disponible para hacerlo es de 20 minutos

\section{Fases}

Acción: Facilitar a cada alumno una hoja de trabajo donde se encuentre el problema planteado, así como la tabla de registro de los paquetes. Proporcionarles los cuatro paquetes de palillos por bina para que organicen $y$ registren. Dar la indicación de que pueden iniciar con la solución de la problemática.

Mientras se encuentren operando, recorrer los pasillos de las filas para corroborar de que se encuentren resolviendo el problema, así como revisar que no haya inconveniencias con el material. 
Formulación: Cuando hayan terminado, solicitar que algunos de los alumnos que expresen sus resultados, de acuerdo al conteo que hicieron y sobre la forma de identificar cuando un número es más grande que el otro. Revisar si hay formas variadas para solucionar el problema.

Validación: Los alumnos que hayan expresado sus resultados, escribirlos en el pizarrón (solución de las preguntas). El orden de participación se dará de acuerdo a la gradualidad de los procedimientos (de menor a mayor) para que se discuta y vayan formulando sus conjeturas.

Dar una orientación para su explicación a partir de las preguntas ¿qué hicieron para saber cuál vale más?, ¿en qué se fijaron para escribir sus respuestas?

De acuerdo a sus participaciones, revisar las diferencias y similitudes en los procedimientos y soluciones, así como llevar a la pregunta de ¿cuál es la forma correcta para saber cuál número es más grande? De manera que puedan elegir entre las que se han expresado la que se crea más conveniente.

Institucionalización: De acuerdo con lo que se haya comentado, retomar de nueva cuenta la situación inicial, así como los datos específicos, lo que los alumnos han considerado para resolver el problema.

Cuestionar sobre la respuesta que se tiene del problema, en el número que es mayor ¿cuánto vale el número de la izquierda (señalarlo) y el de la derecha (señalarlo)? ¿qué significado tiene el número de la izquierda? Esto para guiar a la importancia de considerar que, en los números de dos cifras, el de la izquierda es el de las decenas y el derecho de las unidades.

Revisar los números de los otros paquetes para comparar el número de decenas, de manera que den cuenta de que entre sea más grande el número de la izquierda, el número es mayor.

Finalmente si se tuviera el número 33 ¿cuánto vale cada número? para que quede claro cuál es el valor de acuerdo a la posición que tiene.
De esta manera se trabaja el valor posicional, fortalece el conteo de 10 en 10 , agrupamiento, identificación de las decenas y unidades, posicionalidad en números de dos cifras, fortalecer la comparación, secuencia y orden.

\section{Experimentación.}

Esta fase fue llevada a cabo en la sesión que se planificó. Tuvo una duración de aproximadamente una hora y media, en ella, se utilizaron instrumentos para recolectar datos importantes sobre los sucesos de la clase: registro de clase, productos finales y video.

\section{Análisis a posteriori y evaluación.}

Para realizar el presente análisis es necesario revisar lo que desde un momento inicial se tomó en cuenta, para hacer un contraste con los resultados que se obtuvieron. En este sentido, la indagación que se realizó en el aspecto del aprendizaje que se pretendía enseñar y las características de los alumnos, fueron consideradas para la planificación de las sesiones y con ello las actividades que se realizaron. Desde términos generales, se puede decir que la obtención de resultados y su análisis arrojó resultados favorables, donde los se distingue el avance de los alumnos en cuanto al concepto de número y su consolidación. Las evidencias que dan prueba de ello y el análisis respectivo que se tiene a esta fase se revisará de manera detallada en los resultados de la investigación. La evaluación viene conjunta con los resultados. Se expresa concretamente en un cuadro de fortalezas y debilidades. De éste y su análisis del se toma en cuenta los aspectos donde es necesario replantear las acciones convenientes para que haya una mejora de la práctica docente.

\section{Perspectiva Teórica}

Michele Artigue, hace mención del término de ingeniería didáctica como "una forma de trabajo didáctico equiparable con el trabajo del ingeniero quien, para realizar un proyecto determinado, se basa en los conocimientos científicos de su dominio y acepta someterse a un control de tipo científico" (1995, pág. 33), a partir de esto, la ingeniería didáctica viene cumpliendo con dos funciones, en las producciones de enseñanza, así como metodología de investigación específica. 
La ingeniería didáctica como metodología de investigación se caracteriza por ser "un esquema experimental basado en las realizaciones didácticas en clase, es decir, sobre la concepción, realización, observación y análisis de secuencias de enseñanza" (Artigue, 1995, pág. 36) pero a la vez, se puede clasificar en alguno de los dos niveles que son: microingeniería (local-aula) y macro-ingeniería (varias micro-ingenierías, enseñanzaaprendizaje).

La ingeniería didáctica tiene un enfoque "en el registro de los estudios de caso y cuya validación es en esencia interna, basada en la confrontación entre el análisis a priori y a posteriori" (Artigue, 1995, pág. 37) es aquí donde se considera particular las investigaciones como ingeniera didáctica, no tanto por los objetivos, sino más bien "por las características del funcionamiento metodológico" (Artigue, 1995, pág. 38). Las características de esta metodología se encuentran definidos en sus cuatro momentos esenciales o fases: análisis preliminar, concepción y análisis a priori de las situaciones didácticas de la ingeniería, experimentación y finalmente de análisis a posteriori y evaluación y que para los intereses de investigación del presente artículo, significaron una oportunidad de análisis en el proceso de construcción de la noción del número en los primeros grados de educación primaria. También se encuentra una relación intrínseca en las intenciones prioritarias de los principios de la ingeniería didáctica y la investigación acción. Para este artículo no se piensa en fundamentar esta última idea, sólo se vislumbra el uso de ambas por la posibilidad de análisis ante un objeto de investigación como es la construcción de la noción de número en educación primaria.

\section{Resultados de la investigación}

Para que se lleve a cabo la construcción del conocimiento, la preparación previa que el docente requiere, estriba no sólo en la organización de las actividades que se llevarán a cabo durante la clase, sino desde la parte conceptual y didáctica. Es necesario que el docente "disponga, en tanto que profesional de la enseñanza, de herramientas y técnicas profesionales que le permitan abordar la enseñanza de las matemáticas con cierta garantía" (Chamorro, 2003, pág. 70).
En esta ocasión, pudo distinguirse esta preparación a partir de la investigación teórica que se enmarca en apartados anteriores, y más específica en el diseño y aplicación de la ingeniería (Análisis a priori), dado que presenta los conceptos básicos que se desean desarrollar en los alumnos, oportunidades, limitaciones, conocimiento de los alumnos, y las estrategias utilizadas. Otro aspecto relevante dentro de esta preparación son los momentos por los que se transita en la clase, son vitales para el proceso de construcción de un conocimiento. De este modo, la secuencialidad de las actividades tiene un porqué que permite al alumno conducirse para lograr el aprendizaje esperado. La clase planteada dentro de la ingeniería fue trazada bajo la dirección de los momentos de la Teoría de las Situaciones Didácticas, presentando en cada clase una situación problema, acompañada por una fase de acción, formulación, e institucionalización.

Un ejemplo claro que muestra esta preparación, es en la intervención del docente en la institucionalización. En el siguiente fragmento de registro, el docente cierra la sesión, su mediación es acorde con el lenguaje de los alumnos y la situación problema planteada:

328.Ma: entonces, el primero es el que nos dice 329.los montoncitos de diez y el 330.otro lo que nos sobra. Si tenemos noventa y 331.nueve ¿Cuántos montoncitos tenemos? (Registro 1)

Aunque en este momento aún no se formaliza el concepto específico de unidades y decenas, su esencia en los agrupamientos da forma a que los alumnos vayan identificando el valor posicional, que es lo que se pretende desde las intenciones didácticas propuestas por el programa de estudios. Cuando, por lo contrario, se es ajeno de lo que se pretende llegar, se suele caer en la improvisación no regulada o simple cambio de actividad que no favorece en el proceso de aprendizaje. Al considerar los conocimientos previos de los alumnos, si bien está relacionado con la tarea del docente, pues "conocer lo que saben la mayoría de los alumnos de un grupo permite al docente decidir a partir de qué momento o de qué aspectos debe partir su intervención" (SEP, 2011, pág. 247), con estas bases, el docente adopta condiciones para que las clases sean favorables. 
Un ejemplo de este momento puede verse reflejado en el siguiente registro del desarrollo de la ingeniería, donde tiene como intención didáctica que los alumnos resuelvan problemas que implican analizar la posición de las cifras de los números involucrados en este momento se distingue el concepto de número mayor para diferenciarlo de uno menor:

10.Ma: ¿Cuál de los dos es más grande?

11.Aos: el ochenta y nueve número?

12.Ma:¿Por qué es más grande ese

13.Ao: porque tiene más números

14.Ma: ¿Tiene más números?

15.Aa: porque tiene el ocho, porque tiene el ocho 16.y... el ocho

17.Ma: ¿Porque tiene el ocho?

18.Ao: $y$ un nueve

19.Ma: ¿Y por eso es más grande?

20.Ao: porque tiene más

21.Ma: ¿Más qué?

22.Ao: Mas bolitas o más de eso (Registro 1)

Aquí, los alumnos tienen ya la noción de inclusión del número, como lo menciona en el renglón 13, al considerar que se incluyen dentro de ese número más números pequeños; en segunda cuenta, cuando menciona que tiene "más bolitas o más de eso" da cuenta nuevamente de que identifica que el número más grande corresponde al conjunto que tiene más elementos. En las dos respuestas se identifican sus saberes previos, pero no formalizados dentro del concepto de valor posicional, que permite con solo observar la posición de los números identificar cuál número es mayor.
Los conocimientos previos además, son parte importante dentro de la construcción del conocimiento, ya que éstos "no se producen a partir de la nada, su elaboración está sometida a adaptaciones, rupturas y a reestructuraciones, a veces radicales, de los conocimientos anteriores" (Chamorro, 2003, pág. 45) y para dar cuenta de lo que se ha construido, es necesario conocer lo que se tiene y el cambio o transformación al que se ha llegado.

\section{La Situación problema.}

Durante el desarrollo de la ingeniería didáctica, se plantearon diversas situaciones problemáticas en el aula adaptadas al medio en el que se indagó desde los análisis preliminares. En cada clase aplicada se diseñó una según el contenido y la intención didáctica que se pretendía trabajar, dado que el Programa de estudios 2011 enmarca que "las situaciones planteadas deberán implicar justamente los conocimientos y habilidades que se quieren desarrollar" (2011, pág. 75) por lo que también va acompañada de instrucciones que definen parte de la actividad e indirectamente se encuentra inmerso el reto cognitivo al que se enfrentaron los alumnos. Una de ellas es la siguiente:

La mamá de Jorge está haciendo bolos para regalarlos en el cumpleaños de su hijo. Dice que serán bolos diferentes, y necesita acomodarlos de acuerdo al número de dulces.

Ayúdale a la mamá de Jorge a contar los dulces de los bolos, escribir cuántos tiene cada bolsa y ordenarlos del que tenga menos al que tenga más.

Su diseño, debe ser basado "de tal modo tal que el alumno pueda aceptarlos, deben lograr, por su propio movimiento en que el alumno acepta el problema como suyo y aquel en que se produce su respuesta" (Brousseau, 2007, pág. 31) y este problema da cuenta de ello desde el hecho de que fue resuelto por los alumnos.

Otro punto importante resaltar es que la situación problemática le da la libertad al alumno para experimentar y plantear sus propios procedimientos, tal como lo menciona el programa de estudios 
Se trata también de que los alumnos sean capaces de resolver un problema utilizando más de un procedimiento, reconociendo cuál o cuáles son más eficaces, o bien, que puedan probar la eficacia de un procedimiento al cambiar uno o más valores de las variables o el contexto del problema para generalizar procedimientos de resolución (SEP, 2011, pág. 278)

A partir de esta aclaración, se da un giro diferente, dado que en el último párrafo de la situación problema, más que ser parte de las instrucciones dentro de la consigna, está siendo el procedimiento que los alumnos deben hacer. Por otro lado, hay que mencionar que el centro del aprendizaje en esa clase era el orden de los números de dos cifras, sin embargo, el buscar el procedimiento de ordenarlos, es parte también de discutir y conjeturar los procedimientos más convenientes.

La experiencia del conocimiento de los números.

Los contenidos específicos que se consideraron fueron referidos al inicio del análisis del valor posicional, la sucesión oral y escrita de números hasta el 100 y la descomposición con sumas con un sumando que se repite y algo más. Todos desarrollados a partir de una situación problema.

Desde un principio, la ingeniería didáctica permite tener "un abanico de útiles, nada triviales, que un profesional tiene que conocer y controlar para ser eficaz en su trabajo como enseñante" (Chamorro, 2003, pág. 82) esta herramienta docente lleva desde su preparación, parte ganada por todas las atenciones que ha previsto para la construcción de un saber en juego.

Durante el desarrollo de la ingeniería, así como fue posible observar las dificultades en cuanto al orden e interés de los alumnos, también fueron distinguidos avances que sin duda marcaron el camino que significaba aprender y fortalecer la noción del número. A partir de la observación se revisó durante la clase, en la mayoría de los alumnos se tuvo un avance, pero no en el mismo aspecto, algunos en el aumento de rango en los números oral y escrito, en la facilidad para contar, cálculo mental, entre otros.
Considerando también el ritmo de aprendizaje de cada uno, la capacidad y la disposición y actitud en la clase. Entre ellos, se encuentran otros que resaltaron y a continuación se presentan. La primera de las clases dentro de la ingeniería didáctica se trabajó con el inicio del análisis del valor posicional, específicamente manipularon palillos de madera agrupándolos en decenas. Para muchos alumnos pudo haber sido una actividad casual que ya habían hecho en otras actividades, sin embargo, el hecho de que ellos la utilicen como estrategia dentro de su proceso es lo que le da sentido a todo.

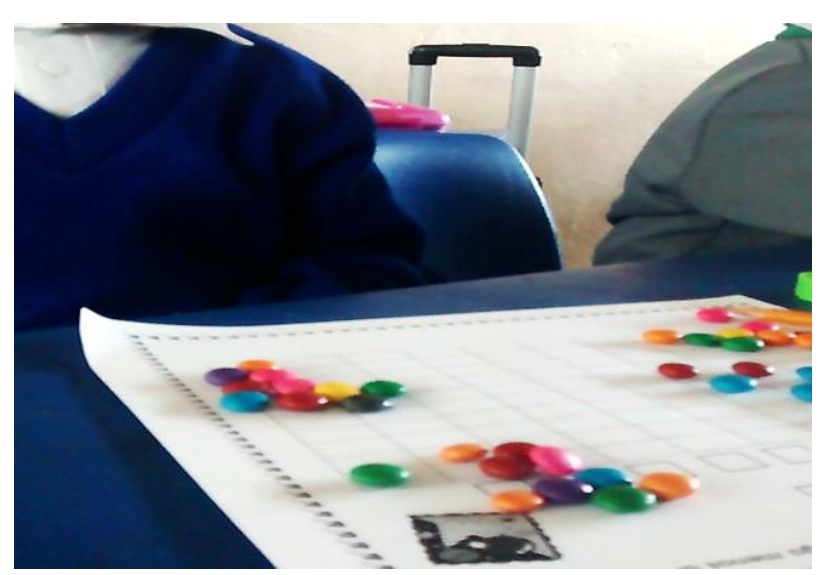

Figura 1 Alumnos agrupando dulces para contar Fuente: propia

Durante la segunda clase de la ingeniería, donde se hizo presente nuevamente el conteo, para comparar los bolos y ordenarlos de menor al mayor, uno de los alumnos utilizó particularmente una estrategia que puede distinguirse en la ilustración 37, ahora su conteo no fue a partir de la correspondencia uno a uno, como los demás, sino que además los agrupó en decenas. Por el número de dulces que le habían tocado, fue sin duda la forma más conveniente de contarlos. Además de que estaba aplicando los conocimientos adquiridos a partir del valor posicional que se revisó en la clase pasada. Otro ejemplo que fue relevante, también identificado en esta sesión fue con una alumna donde al escribir el orden de los números, los clasificó en colores (ilustración 2) por lo que había diez colores diferentes, y los números siguientes en la columna debería corresponder con el primer número (ej. Donde está el seis también debe ir el 16-26-36-46, etc.). De este modo, se puede decir que se cumple con la expectativa del docente, donde los alumnos "aprovechen lo que ya saben y avancen en el uso de técnicas y razonamientos cada vez más eficaces” (SEP, 2011, pág. 247). 


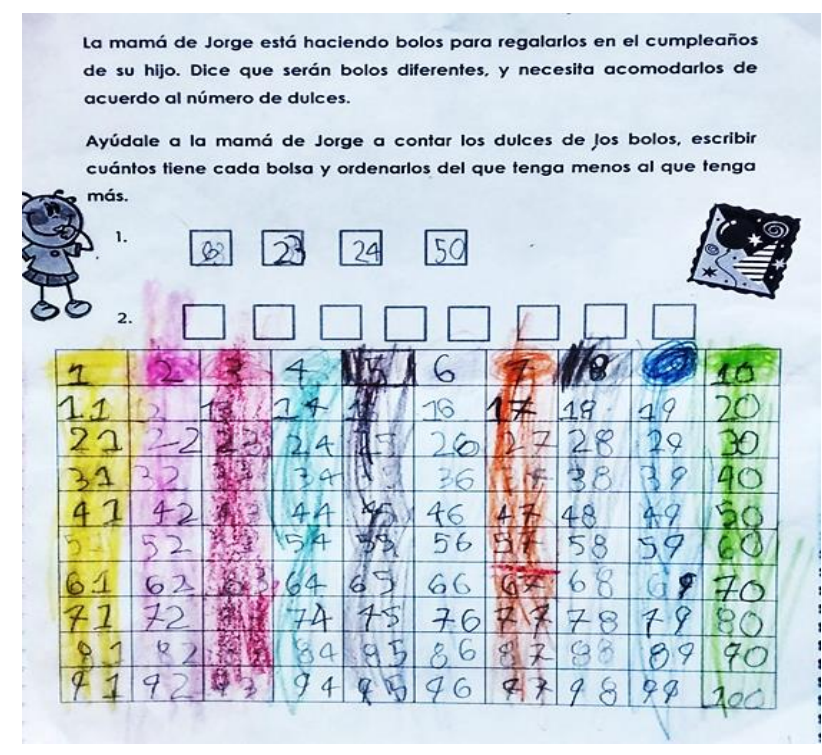

Figura 2 Producto: Clasificación por colores Fuente: propia

\section{Conclusiones}

Se encontraron ideas importantes que es necesario resaltar. Por un lado, es esencial destacar el aspecto de los conocimientos previos, aunque desde el inicio se hace mención de ellos indirectamente en la preparación del docente, no se explicita en lo que consiste. Su participación es más que indispensable en la construcción de cualquier conocimiento en los tres participantes de la relación didáctica: docente, alumno y saber. Son de las bases que van fundamentando la intervención y el logro del proceso de enseñanza - aprendizaje. Cuando se dejan de lado los conocimientos previos, es entendido entonces que no se pretende la construcción del saber, sino la transmisión del mismo, lo que indica un desenfoque del constructivismo que se pretende realizar. Otro punto que se distingue es en la formulación y planteamiento de las situaciones problemas didácticas. Cuando se diseña la situación problema acorde al medio en el que se encuentra el alumno, sin duda el infante se sitúa con su contexto. Sin embargo, es necesario que estas situaciones sean completamente lúdicas, para que en ningún momento se pierda su atención, genere interés por el aprendizaje y le permita tener la seguridad de buscar nuevas oportunidades de resolución. Es preciso recordar que los alumnos se encuentran en la etapa de la infancia, dando valor significativo a sus experiencias y no de igual forma a las actividades que le imponen.
La autonomía de los alumnos es otro de los aspectos que fue distinguida dentro de los resultados. La intervención del docente debe ser la más prudente posible, pues se puede caer en el error de abandonar al alumno en su proceso, o todo lo contrario, limitarlo con acciones o cuestionamientos que pretendan decir lo que el docente quiere oír, pero sin ninguna comprensión. La autonomía apropiada para el proceso de construcción de un conocimiento es precisamente en llevar las fases por las que desde la didáctica de las matemáticas se hacen. Permitir que el alumno experimente, se equivoque, contrastar sus estrategias para que pueda conjeturar sus procedimientos $\mathrm{y}$ resultados.

Para lograr la construcción de la noción del número es fundamental la práctica de actividades relacionadas con las operaciones y principios de la clasificación y la seriación. Su trabajo constante y dedicación al diseñar las clases convenientes para lograr el aprendizaje de este contenido, el desarrollo según el dispositivo y las consideraciones conceptuales, didácticas y análisis de la práctica educativa. Cuando se refleja que los alumnos se apropian de un conocimiento es conforme al cambio que realizan en sí mismos, en su forma de resolver los problemas a los que se enfrentan.

Con lo realizado en la investigación es posible argumentar que las implicaciones conceptuales consisten en conocer los conceptos, principios y acciones inmersos en las operaciones de clasificación y seriación, diferenciando las enfocadas a la construcción del número y el nivel de consolidación al que se pretenda llegar. En las implicaciones didácticas tener presente la didáctica actual, el enfoque propuesto por el plan de estudios vigente, así como conocer la metodología utilizada para llevar a cabo las clases. En este caso, la ingeniería didáctica abarca desde los conocimientos previos necesarios conceptuales, didácticos y con referencia a los alumnos, el diseño de las actividades convenientes, la puesta en marcha y el análisis final que revisa el proceso que se ha llevado a cabo.

Acorde a lo que se ha presentado, es inexcusable mencionar los aspectos que quedan como retos o expectativas por desarrollar en siguientes investigaciones. 
Uno de ellos es el hecho de conocer la importancia que tiene el trabajo colaborativo e individual para que se desarrolle la construcción del número, los ambientes de aprendizaje propios para desarrollar las clases exitosas. Otro elemento importante es retomar los puntos que quedaron pendientes en cuestión a las estrategias basadas en las tecnologías de la información y la comunicación, así como revisar cuáles son las estrategias más convenientes para que la construcción del número y su consolidación. Así mismo, se queda la idea de seguir experimentando la ingeniería didáctica en otros temas de estudio, bien de matemáticas o en otra área del conocimiento.

\section{Referencias}

Artigue, M. (1995). Ingeniería Didáctica. En M. Artigue, R. Douady, \& L. Moreno, Ingeniería didáctica en educación matemática. Un esquema para la investigación y la innovación en la enseñanza y el aprendizaje de las matemáticas. (págs. 33-60). Bogotá: Grupo Editorial Iberoamérica.

Block, D., \& Álvarez, A. (1999). Los números en primer grado: cuatro generaciones de situacionesdidácticas. Educación Matemática, 57-76.

Brousseau, G. (2007). Iniciación al estudio de la teoría de las situaciones didácticas. Argentina: Ministerion de educación.

Chamorro, M. (2003). Didáctica de las matemáticas . Madrid: Pearson Educación.

Hernández Sampieri, R., Fernández Collado, C., \& Baptista Lucio, P. (2006). Metodología de la investigación. Cuarta edición. México: McGrawHill.

Latorre, A. (2005). La investigación-acción. conocer y cambiar la práctica educativa. España: Graó.

Lizarde Flores, E. (2016). La construcción del conocimiento especializado del profesor de matemáticas (MTSK) desde el escenario normalista. Entre maestr@s, 16(57), 82-91.

SEP. (2011). Programa de estudios 2011. Guía para el maestro. Educación básica. Primaria. Primer grado. México: Autor 